\title{
Canada will have three-digit suicide prevention hotline by 2023
}

- Cite as: CMAJ 2021 January 18;193:E106-7. doi: 10.1503/cmaj.1095913

Posted on cmajnews.com on December 16, 2020

S ixteen people in Bill Pringle's life have died by suicide - four in the past year alone. The 62-year-old Saskatoon man has himself attempted suicide eight times. He says that easy access to suicide prevention services could make the difference between life and death for many Canadians but accessing help typically isn't easy. "You can call 911, but suicide prevention is not what they do. You can call crisis lines, but you may not have a local number," Pringle says.

A national suicide prevention service aims to change that. The main pillar of the service, backed by $\$ 21$ million in federal funding over the next five years, will be a three-digit crisis number that people can call from anywhere in the country to connect with trained responders near them.

Distress centres across Canada have reported a surge in calls and texts during the coronavirus disease 2019 pandemic, according to Dr. Allison Crawford, chief medical officer for the Canada Suicide Prevention Service. In September, an unprecedented 1 in 10 Canadians reported experiencing suicidal thoughts, up from $2.6 \%$ before the pandemic.

Launched in 2017, the Canada Suicide Prevention Service already operates a 10-digit crisis hotline, as do other organizations across the country. Research shows these crisis services decrease distress in callers and prevent suicide in the short term. "It's one of the most effective ways we have to intervene in a very highrisk population," says Crawford.

However, Pringle and others have noted that long 1-800 numbers can pose a barrier to access. "A person in the throes of a mental health crisis is not going to

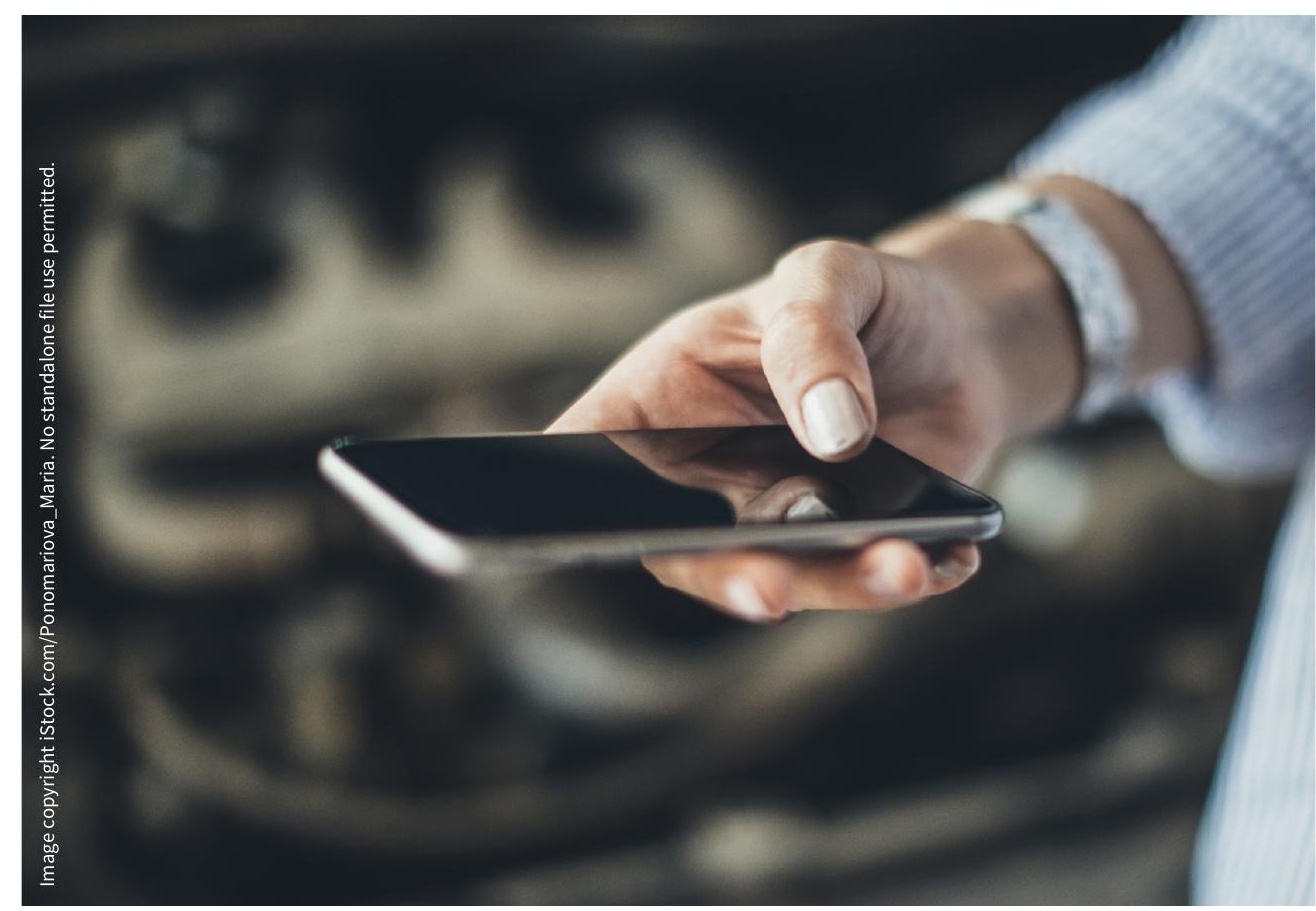

A national suicide prevention service will provide 24/7/365 crisis support.

remember a 10-digit number. It just isn't going to happen," he says.

Pringle says that when a person is either contemplating or attempting suicide, they need immediate help from someone experienced in suicide prevention. "If I had had a dedicated service to reach out to, my attempts might have been fewer and not as severe as they were. We need more tools... this is a step in the right direction."

Parliament passed a unanimous motion in December supporting the creation of a 9-8-8 national suicide prevention number. When fully implemented in March 2023, the Canada Suicide Prevention Service will provide a single access point to $24 / 7 / 365$ crisis support in English and French via phone, text, and online chat. According to the Public Health Agency of Canada, "this includes immediate access to information and resources such as emergency services, referrals, safety plans, and bereavement support."

The Centre for Addiction and Mental Health is rolling out the service in phases in partnership with the Canadian Mental Health Association and Crisis Services Canada. In addition to running the 9-8-8 hotline, the Canada Suicide Prevention Service will network distress centres across the country and develop shared best practices and standards for crisis intervention.

"There's also an advocacy element," Crawford says. "Gold standard" national 
services like the National Suicide Prevention Lifeline in the United States and the United Kingdom's Samaritans charity provide a bigger platform for shared fundraising and communication campaigns. "If you look at something like Bell Let's Talk, the [national] recognition of that has helped, I think, to reduce stigma around mental illness," Crawford says.

Greg Basky, Saskatoon, Sask.

Editor's note: If you or someone you know is currently in crisis, please call 911 or the Canada Suicide Prevention Helpline at 1-833-456-4566, or go to the nearest hospital.

Content licence: This is an Open Access article distributed in accordance with the terms of the Creative Commons Attribution (CC BY-NC-ND 4.0) licence, which permits use, distribution and reproduction in any medium, provided that the original publication is properly cited, the use is noncommercial (i.e., research or educational use), and no modifications or adaptations are made. See: https://creativecommons.org/ licenses/by-nc-nd/4.0/ 